\title{
Birthmark based identification of software piracy using Haar wavelet
}

\begin{abstract}
Piracy of software is an increasing problem of modern day software industry. Piracy of software is the unlawful use of software or part of it without proper permission as described in license agreement. Software piracy is a serious crime but not taken seriously by most people. Preventing software piracy is very important for the growing software industry. Efforts are being made to prevent and detect software piracy. Several techniques have been developed most important of which is software birthmark. The birthmark of a software is the intrinsic properties of software. A recent research shows that a features based software birthmark can be used as a strong mechanism to detect piracy of a software and how much piracy performed has been performed on it. An objective measure is needed to overcome this problem and to compare features based birthmark of a software which efficiently and precisely detect piracy in reproduction of software. The proposed study presents Haar wavelet collocation method for software features (birthmark) to detect piracy. The proposed method gives an exclusive solution for the features based birthmark of software and is then further used for comparisons of birthmark. The results of the proposed study show the effectiveness in terms of accuracy and efficiency to compare the features based software.
\end{abstract}

Keyword: Software piracy detection; Software birthmark; Haar wavelet; Partial differential equation 